\title{
BMJ Open Associations and effects of omega-3 polyunsaturated fatty acids on cognitive function and mood in healthy adults: a protocol for a systematic review of observational and interventional studies
}

\author{
Fiona O' Donovan, ${ }^{\circledR 1,2,3}$ Síle Carney, ${ }^{3,4}$ Jean Kennedy, ${ }^{2}$ Heather Hayes, ${ }^{2}$ \\ Niall Pender, ${ }^{3}$ Fiona Boland, ${ }^{\odot 5}$ Alice Stanton ${ }^{1,6}$
}

To cite: $0^{\prime}$ Donovan F, Carney S, Kennedy J, et al. Associations and effects of omega-3 polyunsaturated fatty acids on cognitive function and mood in healthy adults: a protocol for a systematic review of observational and interventional studies. BMJ Open

2019;9:e027167. doi:10.1136/ bmjopen-2018-027167

- Prepublication history and additional material for this paper are available online. To view please visit the journal (http:// dx.doi.org/10.1136/bmjopen2018-027167).

$\mathrm{FB}$ and $\mathrm{AS}$ contributed equally.

Received 9 0ctober 2018 Revised 27 March 2019 Accepted 21 May 2019

Check for updates

(c) Author(s) (or their employer(s)) 2019. Re-use permitted under CC BY-NC. No commercial re-use. See rights and permissions. Published by BMJ.

For numbered affiliations see end of article.

Correspondence to

Fiona 0' Donovan;

fionaodonovan@rcsi.ie

\section{ABSTRACT}

Introduction The association between long-chain omega-3 polyunsaturated fatty acids (PUFAs), brain health, cognitive function and mood has been the subject of intensive research. Marine-derived omega-3 PUFAs, such as docosahexaenoic acid and eicosapentaenoic acid, are highly concentrated in neuronal membranes and affect brain function. Many studies have found that consumption of omega-3 PUFAs is associated with lower risk of cognitive or mood dysfunction. However, other studies have demonstrated no beneficial effects. There appears to be inconsistent findings from both epidemiological and randomised controlled trial (RCT) studies. The aim of this review is to compile the previous literature and establish the efficacy of omega-3 PUFAs in enhancing cognitive performance and mood in healthy adults.

Methods and analysis Prospective cohort studies, RCTs, controlled clinical trials, controlled before and after studies, interrupted time series with a minimum of 3 months duration will be eligible for inclusion. Studies on healthy adults over the age of 18 , where the intervention/ exposure of interest is omega-3 PUFAs will be included. The outcomes of interest are cognition and mood. Studies will be eligible for inclusion if they measure changes in cognitive function or mood, or the risk of developing cognitive or mood disorders using validated tools and assessments. Relevant search terms and keywords will be used to generate a systematic search in Cochrane Library, MEDLINE, EMBASE, PsycINFO, Cumulative Index to Nursing and Allied Health Literature, Web of Science, Scopus and the grey literature. Two independent reviewers will screen studies for eligibility. Risk of bias in cohort and non-randomised studies will be assessed using the ROBINS-I tool. The Cochrane Collaboration's tool for assessing risk of bias in randomised trials will be used for RCTs. If there are sufficient data, a meta-analysis will be conducted.

Ethics and dissemination This systematic review does not involve primary data collection and therefore formal ethical approval is not required. Results will be disseminated through peer reviewed publications, conference presentations and the popular press. PROSPERO registration number CRD42018080800.
Strengths and limitations of this study

- This systematic review will contribute to the evidence regarding the effectiveness of omega- 3 polyunsaturated fatty acids (PUFAs) on cognitive function and mood in healthy adults.

- The search strategy encompasses seven databases to comprehensively examine the available psychological and medical literature.

- Inclusion of numerous types of study designs will enhance the generalisability of the findings.

- Unlike previous systematic reviews, this study will include only healthy participants and exclude clinical or vulnerable groups.

- The review will examine the impact of omega-3 PUFAs on both cognitive function and mood, both of which affect daily functioning and quality of life.

\section{INTRODUCTION}

Consumption of omega-3 long-chain polyunsaturated fatty acids (PUFAs), from oily fish, has been associated with a number of health benefits involving multiple bodily systems. ${ }^{1}$ Omega-3 PUFAs are highly concentrated in the brain and there is evidence of a link between omega-3 PUFA consumption and brain health and brain function.

Omega-3 PUFAs are purported to exert their beneficial effects in numerous ways. Studies have demonstrated that omega-3 PUFAs play a role in multiple aspects of brain physiology and biological activity, for example,

- Membrane fluidity. Omega-3 PUFAs 'flexible' chemical structure increases the fluidity of neuronal membranes. ${ }^{23}$

- Synaptic function. Related to their structural role in neuronal membranes, omega-3 PUFAs interact directly with membrane bound proteins such as 
enzymes, ion channels and transporters, and glucose transporters thus influencing signal transduction and synaptic activity. ${ }^{45}$

- Inflammation. Omega-3 PUFAs are precursors to anti-inflammatory eicosanoids and induce the synthesis of resolvins and neuroprotectins. ${ }^{67}$

- Cerebral blood flow. Omega-3 PUFAs have well established effects on endothelial function and therefore may impact cerebral blood flow. ${ }^{8}$

- Neurotrophic effects. Omega-3 PUFAs slow the degradation of neural tissue. They exert potent anti-apoptotic effects and help to maintain healthy axons and synaptic structures. ${ }^{9} 10$

Through these and other mechanisms, it is hypothesised that omega-3 PUFA concentrations may influence cognition and mood. ${ }^{11}$

Although mechanistic and epidemiological evidence has provided support for the role of omega-3 PUFAs in enhancing brain function, their effect on human cognitive performance and mood remains unclear. While many studies have demonstrated beneficial effects of omega-3PUFAs on mood or cognitive function, almost as many studies have been negative. ${ }^{12} 13$ These conflicting results could be attributed to sample heterogeneity, methodological inconsistencies or differences between interventions.

Previous systematic reviews have tended to focus on clinical groups, ageing populations and included participants with cognitive impairment or mood disorders. ${ }^{14-22}$ Past systematic reviews have also tended to focus exclusively on randomised controlled trials (RCTs). ${ }^{14-18}{ }^{20-25}$ While RCTs are generally considered the gold standard in establishing efficacy of interventions, they have very tightly controlled procedures and inclusion criteria, meaning that their results can lack external validity. ${ }^{26}$ Moreover, RCTs tend to be short term ( $<6$ months) and focus largely on supplements (as opposed to whole foods).

\section{Importance of clarifying the impact of omega-3 PUFAs on cognitive function and mood}

The pertinence of clarifying the effects of omega-3 PUFAs on brain health is underlined by findings that omega-3 PUFA consumption is low or very low in both developed and developing countries worldwide. ${ }^{27}$ Modern western diets are notably deficient in omega-3 PUFAs. ${ }^{28}$

Brain disorders (mental and neurologic) were estimated to cost $€ 798$ billion in Europe in 2010 . $^{29}$ It is predicted that over 100 million people worldwide will develop dementia by $2050 .{ }^{30}$ Almost $30 \%$ of people report experiencing a common mental disorder in their lifetime. ${ }^{31}$ Given that both cognitive and mood dysfunction have a profound impact on functional outcomes and quality of life, lifestyle modifications that may mitigate the risk of developing these disorders are of the utmost importance.

A better understanding of the role of these nutritional molecules in normal brain function, is therefore of great interest. The aim of this systematic review is to assess the effectiveness of omega-3 PUFA consumption on cognitive function and mood in healthy adults.
METHODS AND ANALYSIS

\section{Protocol details}

The protocol was registered with the International Prospective Register of Systematic Reviews on 8 October 2018 (https://www.crd.york.ac.uk/PROSPERO/display_ record.php?RecordID=80800). It is reported in accordance with the Preferred Reporting Items for Systematic Review and Meta-Analysis (PRISMA) Protocols. ${ }^{32} 33$

\section{Eligibility criteria}

Types of studies

Prospective cohort studies, RCTs, controlled clinical trials (CCTs), controlled before and after studies (CBAs), interrupted time series (ITS) studies with a clearly defined point time when the intervention occurred and at least three data points before and three data points after the intervention. See online supplementary appendix 1 for description of the eligible study types.

\section{Participants}

Our population of interest is healthy participants over the age of 18 years. We will exclude trials that recruit from specific clinical populations (eg, participants with dementia or depression).

\section{Omega-3 PUFA exposures and interventions}

Observational cohort studies will be eligible for inclusion if they assess omega-3 PUFA status in one of the following ways.

- Measuring omega-3 PUFA levels biologically (eg, plasma or erythrocyte levels).

- Calculating omega-3-PUFA levels from dietary reports.

- Calculating the average number of servings of fish per week.

- Calculating the average number of servings of oily fish per week.

We will include intervention studies that administer long-chain omega-3 PUFAs. The long-chain omega-3 PUFA intervention can be made up by any combination of the following types of omega-3 PUFAs: eicosapentaenoic acid (EPA), docosahexaenoic acid (DHA) and docosapentaenoic acid. This may be administered as fish oils, oil capsules, addition of oily fish to diet or as any other alternative omega-3 PUFA dietary intake intervention. Interventions must be a minimum 3 months in duration (this duration was chosen because although supplementation rapidly increases plasma levels of omega-3 PUFAs, kinetic studies have shown that it takes 4-6 months after the start of DHA supplementation to reach new steady state tissue concentrations $^{34}$ ).

\section{Outcome measures}

Cognitive function and mood are the two primary outcomes of interest. Both outcomes synergistically and separately affect daily functioning and quality of life and therefore were deemed of equal importance.

Studies will be eligible for inclusion if they measure changes in cognitive function or the risk of developing cognitive impairment or dementia using validated 
cognitive tests (eg, the Mini Mental State Exam ${ }^{35}$ or the Wechsler Memory Scales ${ }^{36}$ ). Cognition may be quantified using a 'global cognitive function' score, or by measurement of discrete cognitive abilities such as memory or attention.

Likewise, studies will be eligible for inclusion if they measure changes in mood or the risk of developing a mood disorder using validated scales or tests (eg, the Hospital Anxiety and Depression Scale ${ }^{37}$ or Beck's Depression Inventory ${ }^{38}$ ).

A secondary outcome of interest are adverse events.

\section{Search strategy for the identification of relevant studies}

A search will be conducted in collaboration with academic librarians on the following electronic databases: Cochrane Library, MEDLINE, EMBASE, PsycINFO, Cumulative Index to Nursing and Allied Health Literature, Web of Science and Scopus. The search strategy will include terms relating to or describing the exposure or intervention (intake of omega-3 PUFAs) and outcomes of interest (cognitive function and mood). Search terms will include 'omega three fatty acid', 'fish oil', 'eicosapentaenoic acid', 'docosahexaenoic acid', 'cognition', 'memory', 'dementia', 'neuropsychological test', 'mood', 'mental health' and 'depression'. Search strategies will be altered to suit each specific database. Current search strategies are outlined in online supplementary appendix 2 .

Further searches will involve journal hand searches, searching reference lists of studies which meet inclusion criteria, identification of grey literature (eg, via online resources such as www.openthesis.org and www.opengrey. $\mathrm{eu})$.

We will only include studies published in the last 20 years, since 1998. This cut-off was selected because it was after this period that the benefits of omega-3PUFAs for human health became widely appreciated and researched. ${ }^{39}$ There will be no restrictions based on language. Searches will be saved to EndNote X7 to create a master file of all references.

\section{Screening of the studies}

Duplicates will be identified and removed using EndNote X7's 'find duplicates' function. Initially, titles and abstracts of identified studies will be reviewed by one reviewer (FOD) to determine potential eligibility. Full texts of potentially eligible studies will be obtained and reviewed by two independent researchers (FOD and SC). Any disagreements or disparities will be resolved by discussion, with reference to a third reviewer when necessary.

\section{Data extraction}

One reviewer (FOD) will extract data using a tailored data extraction form and the information extracted with include information related to: (a) study citation, (b) details of intervention, (c) participants, (d) setting, (e) study design and (f) primary and secondary outcomes.

If a study presents missing, unclear, or incompletely reported data, we will attempt to contact the study authors to obtain the data. The extent of missing data will be documented in the extraction form.

\section{Risk of bias}

Two review authors (FOD and SC) will independently assess the risk of bias of each included study.

Bias in cohort and non-randomised studies will be evaluated using the ROBINS-I tool. Each study will be evaluated against the seven domains of bias signalled by the ROBINS-I, arriving at an overall bias judgement. We will resolve disagreements by consensus or a third review author (AS or FB) will be asked to resolve disagreements if necessary.

Similarly, RCTs will be evaluated against the criteria described in the Cochrane risk of bias tool. We will explicitly judge each of these criteria using: low risk of bias, high risk of bias or unclear risk of bias (either lack of information or uncertainty over the potential for bias). Again, disagreements will be resolved by discussion and consultation with a third review author (AS or FB) if necessary.

Risk of bias will be assessed at the study level. No studies will be excluded as a result of the risk of bias assessments.

\section{Data synthesis}

Randomised and observational studies will be reported separately. Where appropriate, a meta-analysis will be conducted using Cochrane Review Manager software ${ }^{40}$ or Stata V.15. ${ }^{41}$

For continuous variables, standardised mean differences (SMD) will be estimated. Each distinct cognitive or mood domain outcome will be evaluated separately (eg, attention distinct from memory, depression distinct from anxiety). SMD is calculated by finding the mean difference (change scores) of the continuous variables of interest (eg, attention, memory) between the intervention and control groups at the end of the study and dividing it by the pooled SD. SMD is a no-unit measure of effect size that allows for comparison of intervention effects across studies where the outcomes have been measured on different scales. Conventionally, SMD values of 0.2 , 0.5 and 0.8 are taken as small, medium and large effect sizes, respectively. ${ }^{42}$ Dichotomous outcomes, if any, will be presented as risk ratios or OR with 95\% CIs. Studies will be pooled together and analysed using a random-effects model to obtain the summary effect estimate, $95 \%$ CI and $\mathrm{p}$ value. Studies using binary and continuous outcomes will be analysed separately.

\section{Assessment of heterogeneity}

Heterogeneity between studies will be explored through visual inspection of the forest plots and using the $\mathrm{I}^{2}$ statistic. We will interpret an $\mathrm{I}^{2}$ value of $0 \%$ is an indication of no observed inconsistency/heterogeneity, $30 \%-60 \%$ as may represent moderate heterogeneity, $50 \%-90 \%$ as may represent substantial heterogeneity and $75 \%-100 \%$ considerable heterogeneity. ${ }^{43}$ Reasons behind any substantial $(\geq 50 \%)$ heterogeneity will be 
explored by performing subgroup and sensitivity analyses as described below. ${ }^{44}$

\section{Dealing with incomplete information}

Where required study data are incomplete or clarifications are needed, authors will be contacted. Following contacting authors, if data are still missing, estimation of SDs will be done by borrowing SDs from other studies included in this meta-analysis. ${ }^{45}$

\section{Assessment of publication bias}

If 10 or more trials are included in the meta-analysis, a funnel plot and Egger's test will be used to assess publication bias. ${ }^{46}$

\section{Analysis of subgroups or subsets}

If sufficient data are available from the included studies, the following subgroup analyses will be conducted:

- The duration of the intervention (3-6 months vs 6 months + ).

- Food studies and supplement studies.

- Different outcome measures (eg, Montreal Cognitive Assessment vs Mini Mental State Exam).

- The formulation of the intervention (ie, quantity of DHA and EPA, quality of supplement).

- Males and females.

- Apolipoprotein E 4 (APOE4) carriers and non-carriers.

- Older adults and younger adults ( $<65$ vs $\geq 65$ years).

Sensitivity analysis

The following sensitivity analyses will be conducted:

- Excluding high risk of bias studies, as classified under 'risk of bias' assessment.

- Excluding outcomes with imputed values.

Should a meta-analysis be not suitable, a tabulation and narrative summary of populations, interventions and outcomes will be completed.

\section{Summary of findings table and quality assessment}

Key outcomes will be presented in a 'Summary of findings' table.

\section{Presenting and reporting of results}

Results will be reported in line with the PRISMA statement. $^{4748}$

\section{Public and patient involvement}

Patients and the public were not involved in the development and design of this study.

\section{ETHICS AND DISSEMINATION}

This systematic review will collect secondary data only and therefore does not require formal ethical approval. Findings will be disseminated through peer reviewed publications, conference presentations and the popular press.

\section{CONCLUSION}

This systematic review will provide an overview of high quality literature that has examined the role of omega-3 PUFAs in cognitive function and mood in healthy adults. By including prospective cohort studies, RCTs, CCTs, CBAs and ITSs this review can incorporate diverse settings, participants, exposures and delivery of interventions, enabling a richer understanding of the relationship between omega-3-PUFAs, cognitive function and mood.

Further, where sufficient data are available, we will conduct a meta-analysis. This will generate useful information regarding the expected effect size associated with an increase in omega-3 PUFA intake. Overall, this review will complement the evidence base on the brain health benefits of omega-3 PUFAs.

\section{Author affiliations}

${ }^{1}$ Molecular and Cellular Therapeutics, Royal College of Surgeons in Ireland, Dublin, Ireland

${ }^{2}$ Food Innovation Department, Devenish Nutrition Ltd, Belfast, UK

${ }^{3}$ Department of Psychology, Beaumont Hospital, Dublin, Ireland

${ }^{4}$ Academic Unit of Neurology, Trinity College Dublin, Dublin, Ireland

${ }^{5}$ Division of Population Health Sciences, Royal College of Surgeons in Ireland, Dublin, Ireland

${ }^{6}$ Food Innovation Department, Devenish Nutrition Ltd, Belfast, UK

Acknowledgements Andrew Simpson, Breffni Smith and Paul Murphy from RCSI library contributed to the development of the search strategy.

Contributors All authors, FOD, SC, JK, HH, NP, FB and AS contributed to the conception and development of the study. AS, FB and FOD were primarily responsible establishing the inclusion and exclusion criteria, data extraction criteria, analyses and interpretation procedure. FOD drafted the manuscript, AS and FB provided critical revisions of the paper. FOD is the guarantor of the review. All authors read and approved the manuscript.

Funding The lead reviewer (FOD) is on a PhD programme funded by the Irish Research Council and Devenish Nutrition, grant code: 2016 EBPPG/2016/351.

Competing interests Several team members are affiliated with Devenish Nutrition, a company that is involved in the production of omega-3 PUFA-enriched food products. FOD is a PhD student funded by the Irish Research Council Employment Based Postgraduate scheme in partnership with Devenish Nutrition. HH is Food Innovation Director with Devenish Nutrition. JK is Food Innovation Manager with Devenish Nutrition. AS works with and has ownership interest with Devenish Nutrition.

Patient consent for publication Not required.

Provenance and peer review Not commissioned; externally peer reviewed.

Open access This is an open access article distributed in accordance with the Creative Commons Attribution Non Commercial (CC BY-NC 4.0) license, which permits others to distribute, remix, adapt, build upon this work non-commercially, and license their derivative works on different terms, provided the original work is properly cited, appropriate credit is given, any changes made indicated, and the use is non-commercial. See: http://creativecommons.org/licenses/by-nc/4.0/.

\section{REFERENCES}

1. Ruxton $\mathrm{CH}$, Reed SC, Simpson MJ, et al. The health benefits of omega-3 polyunsaturated fatty acids: a review of the evidence. $J$ Hum Nutr Diet 2004;17:449-59.

2. Bourre JM, Dumont O, Piciotti M, et al. Essentiality of omega 3 fatty acids for brain structure and function. World Rev Nutr Diet 1991:66:103-17.

3. Yehuda S, Rabinovitz S, Mostofsky DI. Essential fatty acids are mediators of brain biochemistry and cognitive functions. J Neurosci Res 1999;56:565-70. 
4. Dyall SC. Long-chain omega-3 fatty acids and the brain: a review of the independent and shared effects of EPA, DPA and DHA. Front Aging Neurosci 2015;7:52.

5. Innis SM. Dietary (n-3) fatty acids and brain development. J Nutr 2007;137:855-9.

6. Calder PC. Polyunsaturated fatty acids and inflammation. Prostaglandins Leukot Essent Fatty Acids 2006;75:197-202.

7. Farooqui AA, Horrocks LA, Farooqui T. Modulation of inflammation in brain: a matter of fat. J Neurochem 2007;101:577-99.

8. Wang Q, Liang X, Wang L, et al. Effect of omega-3 fatty acids supplementation on endothelial function: a meta-analysis of randomized controlled trials. Atherosclerosis 2012;221:536-43.

9. Bazan NG. Omega-3 fatty acids, pro-inflammatory signaling and neuroprotection. Curr Opin Clin Nutr Metab Care 2007;10:136-41.

10. Calon F, Lim GP, Yang F, et al. Docosahexaenoic acid protects from dendritic pathology in an Alzheimer's disease mouse model. Neuron 2004;43:633-45.

11. Muldoon MF, Ryan CM, Yao JK, et al. Long-chain omega-3 fatty acids and optimization of cognitive performance. Mil Med 2014;179(11 Suppl):95-105.

12. Bozzatello P, Brignolo E, De Grandi E, et al. Supplementation with omega-3 fatty acids in psychiatric disorders: a review of literature data. J Clin Med 2016:5:67.

13. Pusceddu MM, Kelly P, Stanton C, et al. N-3 polyunsaturated fatty acids through the lifespan: implication for psychopathology. Int $J$ Neuropsychopharmacol 2016;19:pyw078.

14. Abubakari AR, Naderali MM, Naderali EK. Omega-3 fatty acid supplementation and cognitive function: are smaller dosages more beneficial? Int J Gen Med 2014;7:463-73.

15. Cooper RE, Tye C, Kuntsi J, et al. Omega-3 polyunsaturated fatty acid supplementation and cognition: a systematic review and metaanalysis. J Psychopharmacol 2015;29:753-63.

16. Burckhardt $M$, Herke M, Wustmann T, et al. Omega-3 fatty acids for the treatment of dementia. Cochrane Database Syst Rev 2016;4:Cd009002.

17. Sydenham E, Dangour AD, Lim W-S. Omega 3 fatty acid for the prevention of cognitive decline and dementia. Cochrane Database Syst Rev 2012;6:CD005379.

18. Mazereeuw G, Lanctôt KL, Chau SA, et al. Effects of $\omega-3$ fatty acids on cognitive performance: a meta-analysis. Neurobiol Aging 2012;33:1482.e17-29.

19. Issa AM, Mojica WA, Morton SC, et al. The efficacy of omega-3 fatty acids on cognitive function in aging and dementia: a systematic review. Dement Geriatr Cogn Disord 2006;21:88-96.

20. Canhada S, Castro K, Perry IS, et al. Omega-3 fatty acids supplementation in Alzheimer's disease: a systematic review. Nutr Neurosci 2018;21:529-38.

21. Appleton KM. Omega-3 fatty acids for depression in adults. Cochrane Database Syst Rev 2015;11:Cd004692.

22. Bae JH, Kim G. Systematic review and meta-analysis of omega-3fatty acids in elderly patients with depression. Nutr Res 2018;50:1-9.

23. Forbes SC, Holroyd-Leduc JM, Poulin MJ, et al. Effect of nutrients, dietary supplements and vitamins on cognition: a systematic review and meta-analysis of randomized controlled trials. Can Geriatr J 2015;18:231-45.

24. Jiao J, Li Q, Chu J, et al. Effect of n-3 PUFA supplementation on cognitive function throughout the life span from infancy to old age: a systematic review and meta-analysis of randomized controlled trials. Am J Clin Nutr 2014;100:1422-36.

25. Rangel-Huerta OD, Gil A. Effect of omega-3 fatty acids on cognition: an updated systematic review of randomized clinical trials. Nutr Rev 2018;76:1-20.

26. Rothwell PM. Factors that can affect the external validity of randomised controlled trials. PLoS Clin Trials 2006;1:e9.
27. Stark KD, Van Elswyk ME, Higgins MR, et al. Global survey of the omega-3 fatty acids, docosahexaenoic acid and eicosapentaenoic acid in the blood stream of healthy adults. Prog Lipid Res 2016;63:132-52.

28. Micha R, Khatibzadeh S, Shi P, et al. Global, regional, and national consumption levels of dietary fats and oils in 1990 and 2010: a systematic analysis including 266 country-specific nutrition surveys. BMJ 2014;348:g2272

29. Olesen J, Gustavsson A, Svensson M, et al. The economic cost of brain disorders in Europe. Eur J Neurol 2012;19:155-62.

30. Alzheimer's Disease International, Policy Brief for G8 Heads of Government. The Global Impact of Dementia 2013-2050. London, UK, 2013.

31. Steel Z, Marnane C, Iranpour C, et al. The global prevalence of common mental disorders: a systematic review and meta-analysis 1980-2013. Int J Epidemiol 2014;43:476-93.

32. Moher D, Shamseer L, Clarke M, et al. Preferred reporting items for systematic review and meta-analysis protocols (PRISMA-P) 2015 statement. Syst Rev 2015;4:1.

33. Shamseer L, Moher D, Clarke M, et al. Preferred reporting items for systematic review and meta-analysis protocols (PRISMA-P) 2015: elaboration and explanation. BMJ 2015;349:g7647.

34. Arterburn LM, Hall EB, Oken H. Distribution, interconversion, and dose response of $\mathrm{n}-3$ fatty acids in humans. Am J Clin Nutr 2006;83:1467S-76.

35. Folstein MF, Folstein SE, McHugh PR. "Mini-mental state". A practical method for grading the cognitive state of patients for the clinician. J Psychiatr Res 1975;12:189-98.

36. Drozdick LW. The Wechsler Adult Intelligence Scale-Fourth Edition and the Wechsler Memory Scale-Fourth Edition, in Contemporary intellectual assessment: Theories, tests, and issues. 3rd ed. New York, NY, US: Guilford Press, 2012:197-223.

37. Zigmond AS, Snaith RP. The hospital anxiety and depression scale. Acta Psychiatr Scand 1983;67:361-70.

38. Beck AT, Steer RA, Brown GK. Manual for the beck depression inventory-II. San Antonio, TX: Psychological Corporation, 1996.

39. Holman RT. The slow discovery of the importance of $\omega 3$ essential fatty acids in human health. J Nutr 1998;128:427S-33.

40. The Cochrane Collaboration,. Review manager (RevMan). Copenhagen: The Nordic Cochrane Centre, 2014.

41. StataCorp. Stata statistical software: release 15. College Station, TX: StataCorp LLC, 2017.

42. Faraone SV. Interpreting estimates of treatment effects: implications for managed care. P T 2008;33:700-11

43. Ryan R. Heterogeneity and subgroup analyses in Cochrane Consumers and Communication Review Group reviews: Planning the analysis at protocol stage Heterogeneity, 2014.

44. Jakobsen JC, Wetterslev J, Winkel P, et al. Thresholds for statistical and clinical significance in systematic reviews with meta-analytic methods. BMC Med Res Methodol 2014;14:120.

45. Furukawa TA, Barbui C, Cipriani A, et al. Imputing missing standard deviations in meta-analyses can provide accurate results. $J$ Clin Epidemiol 2006;59:7-10.

46. Egger M, Davey Smith G, Schneider M, et al. Bias in meta-analysis detected by a simple, graphical test. BMJ 1997;315:629-34.

47. Liberati A, Altman DG, Tetzlaff J, et al. The PRISMA statement for reporting systematic reviews and meta-analyses of studies that evaluate healthcare interventions: explanation and elaboration. BMJ 2009;339:b2700.

48. Moher D, Liberati A, Tetzlaff J, et al. Preferred reporting items for systematic reviews and meta-analyses: the PRISMA statement. Ann Intern Med 2009;151:264. 